\title{
Superfield Formulation for Non-Relativistic Chern-Simons-Matter Theory
}

\author{
YU NAKAYAMA \\ Berkeley Center for Theoretical Physics, University of California, Berkeley, CA 94720, \\ USA.e-mail: nakayama@berkeley.edu
}

Received: 20 February 2009 / Revised: 4 April 2009 / Accepted: 2 June 2009

Published online: 20 June 2009 - (C) The Author(s) 2009. This article is published with open access at Springerlink.com

\begin{abstract}
We construct a superfield formulation for non-relativistic Chern-Simons-matter theories with manifest dynamical supersymmetry. By eliminating all the auxiliary fields, we show that the simple action reduces to the one obtained by taking non-relativistic limit from the relativistic Chern-Simons-matter theory proposed in the literature. As a further application, we give a manifestly supersymmetric derivation of the non-relativistic ABJM theory.
\end{abstract}

Mathematics Subject Classification (2000). 81T60.

Keywords. superfield, non-relativistic field theory, Chern-Simons theory.

\section{Introduction}

The recent advent of the non-relativistic AdS/CFT correspondence [2,21] makes it more important than recognized before to study non-relativistic superconformal gauge theories. It may have realistic applications to strongly coupled condensed matter physics such as high $T_{c}$ superconductor or quantum Hall effects. On the other hand, theoretical understanding of the AdS/CFT correspondence may be enhanced by the comparison of such theoretical predictions with experiments.

In particular, non-relativistic Chern-Simons-matter theories in $(1+2)$ dimension are of special relevance. The Galilean invariance as well as non-relativistic conformal invariance (known as Schrödinger invariance $[3,7,18,19]$ ) can be implemented by directly taking the non-relativistic limit of relativistic Chern-Simons-matter theory in the same dimension $[10,11]$. The construction can be applied to the supersymmetric Chern-Simons-matter theory as well [12], and explicit examples of non-relativistic superconformal field theories have been constructed in this way $[14,16]$. As a particular example, various non-relativistic limits of the M2-brane gauge theory were scrutinized in [15], ${ }^{1}$ which may admit the explicit

${ }^{1}$ Our construction is to deform the original ABJM theory [1], which corresponds to the M2-brane gauge theory on the orbifold space, by adding the supersymmetric mass term [6,9], which corresponds to the introduction of four-form flux, and take the subsequent non-relativistic limit. 
gravity solution that can be studied in the context of non-relativistic AdS/CFT correspondence.

However, in this approach, the supersymmetry is not manifest, and there is no general argument how to guarantee the supersymmetry preserved in the course of taking the non-relativistic limit. Indeed, we have seen several counterintuitive examples that break the supersymmetry $[14,16]$ through this blind, seemingly harmless, non-relativistic limit. Thus, we have to check the supersymmetry of the proposed non-relativistic action by hand, and sometimes we have to add some other terms, like four-fermi terms, to restore the supersymmetry [14]. In particular, it is very cumbersome to verify the dynamical supersymmetry in the component formulation.

Superfield formulation [20] (see e.g. [5,22] for reviews in the relativistic case) is an elegant way to make the supersymmetry transformation manifest. With the superfield formulation at hand, the supersymmetry transformation is nothing but the translation in the superspace, and the construction of the supersymmetric action becomes simply the total integral over the superspace. In this paper, we develop a superfield formulation for the non-relativistic Chern-Simons-matter theory and we propose a manifest superfield form of the action studied in the literatures. See also [4] for another superfield formulation for non-relativistic systems that has a manifest kinematic supersymmetry. The reference is orthogonal to our approach that focuses on the dynamical supersymmetry.

Our construction is minimal in the sense that we only assume the dynamical supersymmetry realized by the algebra

$$
\left\{Q_{2}^{*}, Q_{2}\right\}=2 H,
$$

so we can use our formulation not only to particular Chern-Simons-matter theories but also any other supersymmetric theories involving this algebra: even conventional relativistic supersymmetric field theories could be rewritten in our formulation while some of the Poincaré symmetry would not be manifest. We may also use our formulation to construct a supersymmetric extension of the LifshitzHořava non-relativistic gauge theory [8].

The organization of the paper is as follows. In Section 2, we introduce the superfield formulation of the non-relativistic gauge theory in $(1+2)$ dimension. In Section 3, we re-examine the non-relativistic Abelian ABJM theory in our formulation. In Section 4, we generalize our construction to the non-Abelian case, and re-derived the action for the non-relativistic ABJM theory with 14 supercharges. In Section 5, we conclude the paper with further discussions.

\section{Superfield Formulation}

In this section, we develop a superfield formulation of the non-relativistic Abelian gauge theory coupled with matters. Our goal is to provide a manifest 
supersymmetric action for the non-relativistic Chern-Simons-matter theory introduced in [12].

\subsection{SUPERSPACE AND SUPERFIELDS}

In order to realize the dynamical supersymmetry

$$
\left\{Q_{2}^{*}, Q_{2}\right\}=2 H
$$

in a manifest form, we introduce the superspace $\left(t, x^{i}, \theta, \bar{\theta}\right)$, where $\theta$ and $\bar{\theta}$ are one-component complex Grassmann coordinate. ${ }^{2}$ We can realize the superalgebra (2.1) by the supercharge

$$
\mathcal{Q}=\frac{\partial}{\partial \theta}+i \bar{\theta} \partial_{t}, \quad \overline{\mathcal{Q}}=\frac{\partial}{\partial \bar{\theta}}+i \theta \partial_{t},
$$

which satisfy the anti-commutation relation $\{\mathcal{Q}, \overline{\mathcal{Q}}\}=2 i \partial_{t}$. We also introduce the supersymmetric derivative:

$$
D=\frac{\partial}{\partial \theta}-i \bar{\theta} \partial_{t}, \quad \bar{D}=\frac{\partial}{\partial \bar{\theta}}-i \theta \partial_{t},
$$

which satisfy the anti-commutation relation $\{D, \bar{D}\}=-2 i \partial_{t}$.

The most general superfield $\Sigma\left(t, x^{i}, \theta, \bar{\theta}\right)$ that lives on the superspace can be expanded as

$$
\Sigma=a+\theta b+\bar{\theta} c+\theta \bar{\theta} d
$$

where $a$ and $d$ are complex bosons and $b$ and $c$ are complex one-component fermions. By acting $\epsilon \mathcal{Q}$ and $\epsilon^{*} \overline{\mathcal{Q}}$, the supersymmetry transformation can be read as

$$
\begin{array}{ll}
\delta_{\epsilon} a=\epsilon b, & \delta_{\epsilon^{*}} a=\epsilon^{*} c \\
\delta_{\epsilon} b=0, & \delta_{\epsilon^{*}} b=\epsilon^{*}\left(d-i \partial_{t} a\right) \\
\delta_{\epsilon} c=-\epsilon\left(d+i \partial_{t} a\right), & \delta_{\epsilon^{*} c}=0 \\
\delta_{\epsilon} d=-i \epsilon \partial_{t} b, & \delta_{\epsilon^{*}} d=i \epsilon^{*} \partial_{t} c .
\end{array}
$$

where $\epsilon, \epsilon^{*}$ are Grassmann supersymmetry parameters. We note that the $\theta \bar{\theta}$ component $d$ transforms as a total derivative, which will be crucial to construct supersymmetric actions.

In order to reduce the degrees of freedom contained in a general superfield, constrained superfields will be useful for our studies. ${ }^{3}$ One simple choice is to impose

\footnotetext{
${ }^{2}$ We always work in $1+2$ dimension, so $i=1,2$. By suppressing the $x^{i}$ dependence, most of the superspace formulation in this section is identical to $\mathcal{N}=2$ supersymmetric quantum mechanics which shares (2.1) (see [17] for the original work). Our construction deviates and becomes original when we discuss the non-relativistic kinetic terms and space component of gauge multiplets in later sections.

${ }^{3}$ For a time being, we assume that the top component of the superfields is bosonic. We will later introduce fermionic superfields as well.
} 
the reality condition $\Upsilon=\Upsilon^{*}$ (real superfield): in component, we have

$$
\Upsilon=a+\theta b-\bar{\theta} b^{*}+\theta \bar{\theta} d,
$$

where $a$ and $d$ are now real bosons and $b$ is a complex fermion.

Another simple constrained superfield is a chiral superfield $X$ satisfying $\bar{D} X=0$ : in component, we have

$$
X=x+\theta \chi-\theta \bar{\theta}\left(i \partial_{t} x\right),
$$

where $x$ is a complex boson and $\chi$ is a complex fermion.

Similarly anti-chiral superfield is obtained by requiring the condition $D \bar{X}=0$, whose component form is

$$
\bar{X}=x^{*}-\bar{\theta} \chi^{*}+\theta \bar{\theta} i \partial_{t} x^{*} .
$$

Note that $X$ and $\bar{X}$ are complex conjugate with each other: $X^{*}=\bar{X}$.

\subsection{GAUGE MULTIPLET}

To construct supersymmetric non-relativistic Chern-Simons-matter theories, we begin with a gauge multiplet. It turns out that the gauge multiplet consists of one real superfield $\mathcal{V}$ and one chiral superfield $\mathcal{A}$ (and its complex conjugate $\mathcal{A}^{*}$ ). We will see that $\mathcal{V}$ includes the time component of the gauge field $A_{0}$ and $\mathcal{A}$ includes the space components $A_{i}$.

A real superfield $\mathcal{V}=a+\theta \kappa-\bar{\theta} \kappa^{*}+\theta \bar{\theta} 2 A_{0}$ has a supersymmetric gauge transformation

$$
\mathcal{V} \rightarrow \mathcal{V}+\Lambda+\bar{\Lambda}
$$

with a supersymmetric gauge parameter given by a chiral superfield $\Lambda=\tilde{\Lambda}+\theta \tilde{\lambda}-$ $\theta \bar{\theta}\left(i \partial_{t} \tilde{\Lambda}\right)$. In component, the supersymmetric gauge transformation takes the form

$$
\begin{aligned}
a & \rightarrow a+\tilde{\Lambda}+\tilde{\Lambda}^{*} \\
\kappa & \rightarrow \kappa+\tilde{\lambda} \\
2 A_{0} & \rightarrow 2 A_{0}-i \partial_{t} \tilde{\Lambda}+i \partial_{t} \tilde{\Lambda}^{*} .
\end{aligned}
$$

It is very convenient to use the WZ-gauge, where $a=\kappa=0$. In this gauge, the supersymmetric gauge transformation is nothing but the ordinary gauge transformation for the time component of the gauge field: $A_{0} \rightarrow A_{0}+\partial_{t} \operatorname{Im} \tilde{\Lambda}$.

The chiral superfield $\mathcal{A}=2 A+\theta \lambda-\theta \bar{\theta}\left(2 i \partial_{t} A\right)$ includes a complex combination of the gauge field $A=A_{1}+i A_{2}$. We impose the supersymmetric gauge transformation

$$
\mathcal{A} \rightarrow \mathcal{A}-2 i \partial_{+} \Lambda,
$$


where $\partial_{+}=\partial_{1}+i \partial_{2}$ (and similarly we define $\partial_{-}=\partial_{1}-i \partial_{2}$ ). It reduces to the usual gauge transformation $2 A \rightarrow 2 A+2 \partial_{+}(\operatorname{Im} \tilde{\Lambda})$ in the WZ-gauge. Similarly, the antichiral superfield $\mathcal{A}^{*}=2 A^{*}-\bar{\theta} \lambda^{*}+\theta \bar{\theta}\left(2 i \partial_{t} A^{*}\right)$ has the supersymmetric gauge transformation $\mathcal{A}^{*} \rightarrow \mathcal{A}^{*}+2 i \partial_{-} \bar{\Lambda}$. Note that the "gaugino" $\lambda$ cannot be eliminated in the WZ-gauge, but we will see that it is an auxiliary field in the Chern-Simonsmatter action.

We propose the supersymmetric extension of the Chern-Simons action as

$$
S_{\mathrm{CS}}=-\frac{\kappa}{16} \int \mathrm{d} t d^{2} x d^{2} \theta\left(\left(\mathcal{A}+2 i \partial_{+} \mathcal{V}\right)\left(\mathcal{A}^{*}-2 i \partial_{-} \mathcal{V}\right)+2 \mathcal{V} \partial_{+} \partial_{-} \mathcal{V}\right),
$$

where the convention of the superspace integration is $\int d^{2} \theta(\theta \bar{\theta})=1$. Because of the superspace integration, the supersymmetric variation of the Lagrangian density is a total derivative and the action is invariant under the dynamical supersymmetry. In addition, the supersymmetric gauge transformation of the Lagrangian density is a total derivative as usual in the Chern-Simons theory, so the action is gauge invariant.

In the WZ-gauge, we can evaluate the action (2.12) as

$$
\int \mathrm{d} t d^{2} x\left(\kappa A_{0} F_{12}+\frac{\kappa}{2} \epsilon^{i j} \partial_{t} A_{i} A_{j}-\frac{\kappa}{16} \lambda \lambda^{*}\right) .
$$

This is the conventional form of the Abelian Chern-Simons action in the nonrelativistic form.

\subsection{MATTER MULTIPLET}

Now, we would like to couple the matter multiplets to the Chern-Simons action. For this purpose, we introduce a chiral superfield

$$
\Phi=\phi+\theta \chi-\theta \bar{\theta}\left(i \partial_{t} \phi\right)
$$

The chiral superfield has the supersymmetric gauge transformation

$$
\Phi \rightarrow \mathrm{e}^{-\Lambda} \Phi,
$$

or similarly, the conjugate anti-chiral superfield $\Phi^{*}$ has the supersymmetric gauge transformation

$$
\Phi^{*} \rightarrow \mathrm{e}^{-\bar{\Lambda}} \Phi^{*}
$$

A simple choice of the action

$$
S_{0}=-\frac{1}{2} \int \mathrm{d} t d^{2} x d^{2} \theta \Phi^{*} \mathrm{e}^{\mathcal{V}} \Phi
$$


is gauge invariant and supersymmetric, but it does not contain any kinetic term for $\phi:^{4}$

$$
S_{0}=\int \mathrm{d} t d^{2} x\left(i \phi^{*}\left(\partial_{0}+i A_{0}\right) \phi+\frac{1}{2} \chi^{*} \chi\right),
$$

so we have to introduce other ingredients. For this purpose, we introduce an associated fermionic chiral superfield $\Psi$, and its conjugate $\Psi^{*}$ :

$$
\begin{aligned}
\Psi & =\psi+\theta \rho-\theta \bar{\theta}\left(i \partial_{t} \psi\right) \\
\Psi^{*} & =\psi^{*}+\bar{\theta} \rho^{*}+\theta \bar{\theta}\left(i \partial_{t} \psi^{*}\right),
\end{aligned}
$$

where $\psi$ is a complex fermion and $\rho$ is a complex scalar. The gauge transformation is $\Psi \rightarrow \mathrm{e}^{\Lambda} \Psi$ and $\Psi^{*} \rightarrow \mathrm{e}^{\bar{\Lambda}} \Psi^{*}$.

Now, we impose the supersymmetric covariant constraint between $\Phi$ and $\Psi$ :

$$
D\left(\mathrm{e}^{\mathcal{V}} \Phi\right)=\left(\partial_{-}+\frac{i \mathcal{A}^{*}}{2}\right) \Psi^{*} .
$$

The constraint is consistent with the supersymmetry and the gauge symmetry. In components (in the WZ gauge), we have the following constraint

$$
\begin{gathered}
\chi=\left(\partial_{-}+i A^{*}\right) \psi^{*} \\
-2\left(i \partial_{t}-A_{0}\right) \phi=\left(\partial_{-}+i A^{*}\right) \rho^{*}-\frac{i}{2} \lambda^{*} \psi^{*} .
\end{gathered}
$$

With this constraint, we proposed a supersymmetric action for the nonrelativistic Chern-Simons-matter theory:

$$
S=S_{\mathrm{CS}}-\frac{1}{2} \int \mathrm{d} t \mathrm{~d} x^{2} d^{2} \theta\left(\Phi^{*} \mathrm{e}^{\mathcal{V}} \Phi+\Psi^{*} \mathrm{e}^{-\mathcal{V}} \Psi\right),
$$

where $S_{\mathrm{CS}}$ is given in (2.12). In the $\mathrm{WZ}$ gauge, the component form of the action can be evaluated as

$$
\begin{aligned}
S= & \int \mathrm{d} t \mathrm{~d} x^{2}\left(\kappa A_{0} F_{12}+\frac{\kappa}{2} \epsilon^{i j} \partial_{t} A_{i} A_{j}-\frac{\kappa}{16} \lambda \lambda^{*}+\right. \\
& +i \phi^{*}\left(\partial_{0}+i A_{0}\right) \phi+\frac{1}{2} \chi^{*} \chi+i \psi^{*}\left(\partial_{0}-i A_{0}\right) \psi+\frac{1}{2} \rho^{*} \rho+ \\
& +b\left(\chi-\left(\partial_{-}+i A^{*}\right) \psi^{*}\right)-b^{*}\left(\chi^{*}-\left(\partial_{+}-i A\right) \psi\right)+ \\
& +c\left[\left(i \partial_{t}-A_{0}\right) \phi+\frac{1}{2}\left(\partial_{-}+i A^{*}\right) \rho^{*}-\frac{i}{4} \lambda^{*} \psi^{*}\right]+ \\
& \left.+c^{*}\left[\left(-i \partial_{t}-A_{0}\right) \phi^{*}+\frac{1}{2}\left(\partial_{+}-i A\right) \rho+\frac{i}{4} \psi \lambda\right]\right) .
\end{aligned}
$$

\footnotetext{
${ }^{4}$ This is because our formulation lacks the manifest Galilean invariance.
} 
Here, in the last three lines, we have introduced a fermionic Lagrange multiplier $b$ and a bosonic Lagrange multiplier $c$ to impose the constraint (2.21).

The action is invariant under the dynamical supersymmetry. It is less obvious but it is also invariant under the kinematical supersymmetry

$$
\left\{Q_{1}, Q_{1}^{*}\right\}=2 M, \quad\left\{Q_{1}, Q_{2}^{*}\right\}=P_{-},
$$

where $M$ is the total mass operator and $P_{-}$is the momentum operator. Alternatively speaking, the action has a Galilean invariance, so the commutator $i\left[G_{-}, Q_{2}\right]=-Q_{1}$ guarantees the existence of the kinematical supersymmetry (see [12] for $\mathcal{N}=2$ non-relativistic supersymmetry algebra). Indeed, we will show that the action (2.23) is equivalent to the one proposed in [12] as a non-relativistic limit of $\mathcal{N}=2$ Chern-Simons-matter theory.

\subsection{EQUIVALENCE TO [12]}

In order to show the equivalence to the action proposed in [12], we first use the equation of motions for $\lambda, \rho$ and $\phi$

$$
\begin{aligned}
\frac{\kappa}{16} \lambda-i \frac{c}{4} \psi^{*} & =0 \\
\frac{\rho}{2}+\frac{1}{2}\left(-\partial_{-}+i A^{*}\right) c & =0 \\
i\left(\partial_{t}+i A_{0}\right) \phi+i\left(\partial_{t}+i A_{0}\right) c^{*} & =0
\end{aligned}
$$

to eliminate $\lambda, \rho$ and $\phi$ (as well as $\chi$ from the constraint). Although the last equation in (2.25) is not kinematical but dynamical, it is easy to see that $\phi=-c^{*}$ is the solution.

Substituting them into the action (2.23), we have

$$
\begin{aligned}
S= & \int \mathrm{d} t \mathrm{~d} x^{2}\left(\kappa A_{0} F_{12}+\frac{\kappa}{2} \epsilon^{i j} \partial_{t} A_{i} A_{j}+\right. \\
& +i c^{*}\left(\partial_{0}-i A_{0}\right) c-\frac{1}{2}\left(\partial_{+}+i A\right) c^{*}\left(\partial_{-}-i A^{*}\right) c+ \\
& \left.+i \psi^{*}\left(\partial_{0}-i A_{0}\right) \psi-\frac{1}{2}\left(\partial_{-}+i A^{*}\right) \psi^{*}\left(\partial_{+}-i A\right) \psi+\frac{c^{*} c \psi^{*} \psi}{\kappa}\right)
\end{aligned}
$$

In order to compare the action with that in [12] we further use the Gauss-law constraint

$$
F_{12}=-\frac{1}{\kappa}\left(c^{*} c+\psi^{*} \psi\right)
$$


and rewrite the kinetic terms by using a trick [16] (up to total derivative terms)

$$
\begin{aligned}
-\frac{1}{2}\left(\partial_{+}+i A\right) c^{*}\left(\partial_{-}-i A^{*}\right) c & =-\frac{1}{2}\left(\partial_{i}+i A_{i}\right) c^{*}\left(\partial_{i}-i A_{i}\right)-\frac{F_{12}}{2} c^{*} c= \\
& =-\frac{1}{2}\left(\partial_{i}+i A_{i}\right) c^{*}\left(\partial_{i}-i A_{i}\right) c+\frac{\left(c^{*} c\right)^{2}+c^{*} c \psi^{*} \psi}{2 \kappa} \\
-\frac{1}{2}\left(\partial_{-}+i A^{*}\right) \psi^{*}\left(\partial_{+}-i A\right) \psi & =-\frac{1}{2}\left(\partial_{i}+i A_{i}\right) \psi^{*}\left(\partial_{i}-i A_{i}\right) \psi+\frac{F_{12}}{2} \psi^{*} \psi
\end{aligned}
$$

Then, the action can be transformed into

$$
\begin{aligned}
S= & \int \mathrm{d} t \mathrm{~d} x^{2}\left(\kappa A_{0} F_{12}+\frac{\kappa}{2} \partial_{t} \epsilon^{i j} A_{i} A_{j}+\right. \\
& +i \Phi^{*}\left(\partial_{0}-i A_{0}\right) \Phi-\frac{1}{2}\left(\partial_{i}+i A_{i}\right) \Phi^{*}\left(\partial_{i}-i A_{i}\right) \Phi+ \\
& +i \Psi^{*}\left(\partial_{0}-i A_{0}\right) \Psi-\frac{1}{2}\left(\partial_{i}+i A_{i}\right) \Psi^{*}\left(\partial_{i}-i A_{i}\right) \Psi+\frac{F_{12}}{2} \Psi^{*} \Psi+ \\
& \left.+\frac{\left(\Phi^{*} \Phi\right)^{2}}{2 \kappa}+3 \frac{\Phi^{*} \Phi \Psi^{*} \Psi}{2 \kappa}\right)
\end{aligned}
$$

by renaming $c \rightarrow \Phi$ and $\psi \rightarrow \Psi$. This action is equivalent to the one presented in [12] with $e=1$ and $m=1 .^{5}$

It is invariant under the dynamical supersymmetry:

$$
\begin{aligned}
\delta \Phi & =\frac{i}{\sqrt{2}} \epsilon^{*} D_{+} \Psi \\
\delta \Psi & =-\frac{i}{\sqrt{2}} \epsilon D_{-} \Phi \\
\delta A & =\frac{2}{\sqrt{2} \kappa} \epsilon \Psi^{*} \Phi \\
\delta A^{*} & =-\frac{2}{\sqrt{2} \kappa} \epsilon^{*} \Psi \Phi^{*} \\
\delta A_{0} & =\frac{i}{2 \sqrt{2} \kappa}\left[\epsilon\left(D_{-} \Psi^{*}\right) \Phi+\epsilon^{*}\left(D_{+} \Psi\right) \Phi\right]
\end{aligned}
$$

as well as the kinematical supersymmetry

$$
\begin{aligned}
\delta \Phi & =\sqrt{2} \eta^{*} \Psi \\
\delta \Psi & =-\sqrt{2} \eta \Phi \\
\delta A_{i} & =0 \\
\delta A_{0} & =\frac{1}{\sqrt{2} \kappa}\left(\eta \Psi^{*} \Phi-\eta^{*} \Psi \Phi^{*}\right) .
\end{aligned}
$$

\footnotetext{
${ }^{5} \mathrm{We}$ can easily recover the mass parameter $m$ by considering the constraint $D\left(\mathrm{e}^{\mathcal{V}} \Phi\right)=$ $\frac{1}{\sqrt{m}}\left(\partial_{-}+\frac{i \mathcal{A}^{*}}{2}\right) \Psi^{*}$.
} 


\section{Non-Relativistic Abelian ABJM Theory}

As a simple application of our superfield formulation, we would like to construct the non-relativistic Abelian $(U(1) \times U(1))$ ABJM theory without referring to the relativistic action. We introduce two vector multiplets $\left(\mathcal{V}, \mathcal{A}, \mathcal{A}^{*}\right)$ and $\left(\hat{\mathcal{V}}, \hat{\mathcal{A}}, \hat{\mathcal{A}}^{*}\right)$ together with 4 matter chiral multiplets $\left(\Phi_{A}, \Psi_{A}\right)(A=1, \ldots, 4)$. The supersymmetric gauge transformations are

$$
\begin{gathered}
\mathcal{V} \rightarrow \mathcal{V}+\Lambda+\bar{\Lambda} \\
\mathcal{A} \rightarrow \mathcal{A}-2 i \partial_{+} \Lambda \\
\hat{\mathcal{V}} \rightarrow \hat{\mathcal{V}}+\hat{\Lambda}+\overline{\hat{\Lambda}} \\
\hat{\mathcal{A}} \rightarrow \hat{\mathcal{A}}-2 i \partial_{+} \hat{\Lambda} \\
\Phi_{A} \rightarrow \mathrm{e}^{-\Lambda+\hat{\Lambda}} \Phi_{A} \\
\Psi_{A} \rightarrow \mathrm{e}^{\Lambda-\hat{\Lambda}} \Psi_{A} .
\end{gathered}
$$

The invariant action that we propose for the Abelian non-relativistic ABJM theory is

$$
\begin{aligned}
& S=\int \mathrm{d} t \mathrm{~d}^{2} x d^{2} \theta\left[-\frac{\kappa}{16}\left(\left(\mathcal{A}+2 i \partial_{+} \mathcal{V}\right)\left(\mathcal{A}^{*}-2 i \partial_{-} \mathcal{V}\right)+2 \mathcal{V} \partial_{+} \partial_{-} \mathcal{V}\right)+\right. \\
& +\frac{\kappa}{16}\left(\left(\hat{\mathcal{A}}+2 i \partial_{+} \hat{\mathcal{V}}\right)\left(\hat{\mathcal{A}}^{*}-2 i \partial_{-} \hat{\mathcal{V}}\right)+2 \hat{\mathcal{V}} \partial_{+} \partial_{-} \hat{\mathcal{V}}\right)- \\
& \left.-\frac{1}{2} \Phi_{A}^{*} \mathrm{e}^{\mathcal{V}-\hat{\mathcal{V}}} \Phi_{A}+\Psi_{A}^{*} \mathrm{e}^{-\mathcal{V}+\hat{\mathcal{V}}} \Psi_{A}\right]
\end{aligned}
$$

with the supersymmetric constraint

$$
D\left(\mathrm{e}^{\mathcal{V}-\hat{\mathcal{V}}_{\Phi_{A}}}\right)=\left(\partial_{-}+\frac{i}{2}\left(\mathcal{A}^{*}-\hat{\mathcal{A}}^{*}\right)\right) \Psi_{A}^{*} .
$$

Just exactly as we did in Section 2, we can eliminate all the auxiliary fields in the components:

$$
\begin{aligned}
\frac{\kappa}{16} \lambda-i \frac{c_{A}}{4} \psi_{A}^{*} & =0 \\
\frac{\kappa}{16} \hat{\lambda}-i \frac{c_{A}}{4} \psi_{A}^{*} & =0 \\
\frac{\rho_{A}}{2}+\frac{1}{2}\left(-\partial_{-}+i A^{*}-i \hat{A}^{*}\right) c_{A} & =0 \\
i\left(\partial_{t}+i A_{0}-i \hat{A}_{0}\right) \phi_{A}+i\left(\partial_{t}+i A_{0}-i \hat{A}_{0}\right) c_{A}^{*} & =0
\end{aligned}
$$

where $c_{i}$ are Lagrange multipliers as before. Substituting them back into the action, we obtain 


$$
\begin{aligned}
S= & \int \mathrm{d} t \mathrm{~d} x^{2}\left(\kappa A_{0} F_{12}+\frac{\kappa}{2} \epsilon^{i j} \partial_{t} A_{i} A_{j}-\kappa \hat{A}_{0} \hat{F}_{12}-\frac{\kappa}{2} \epsilon^{i j} \partial_{t} \hat{A}_{i} \hat{A}_{j}+\right. \\
& +i c_{A}^{*}\left(\partial_{0}-i A_{0}+i \hat{A}_{0}\right) c_{A}-\frac{1}{2}\left(\partial_{+}+i A-i \hat{A}\right) c_{A}^{*}\left(\partial_{-}-i A^{*}+i \hat{A}\right) c_{A}+ \\
& \left.+i \psi_{A}^{*}\left(\partial_{0}-i A_{0}+i \hat{A}_{0}\right) \psi_{A}-\frac{1}{2}\left(\partial_{-}+i A^{*}-i \hat{A}^{*}\right) \psi_{A}^{*}\left(\partial_{+}-i A+i \hat{A}\right) \psi_{A}\right)
\end{aligned}
$$

We can further rewrite the action by using the Gauss-law constraint:

$$
F_{12}=\hat{F}_{12}=-\frac{1}{\kappa}\left(c_{A}^{*} c_{A}+\psi_{A}^{*} \psi_{A}\right)
$$

as (up to total derivative terms)

$$
\begin{aligned}
-\frac{1}{2}\left(\partial_{+}+i A-i \hat{A}\right) c_{A}^{*}\left(\partial_{-}-i A^{*}+i \hat{A}^{*}\right) c_{A} & =-\frac{1}{2} D_{i} c_{i}^{*} D_{i} c_{i} \\
-\frac{1}{2}\left(\partial_{-}+i A^{*}-i \hat{A}^{*}\right) \psi_{A}^{*}\left(\partial_{+}-i A+i \hat{A}\right) \psi_{A} & =-\frac{1}{2} D_{i} \psi_{A}^{*} D_{i} \psi_{A}-\frac{F_{12}-\hat{F}_{12}}{2} \psi_{A}^{*} \psi_{A}= \\
& =-\frac{1}{2} D_{i} \psi_{A}^{*} D_{i} \psi_{A}+\frac{F_{12}-\hat{F}_{12}}{2} \psi_{A}^{*} \psi_{A}
\end{aligned}
$$

Finally, by renaming $c_{A} \rightarrow\left(\Phi_{2},-\Phi_{1}, \Phi_{2^{\prime}},-\Phi_{1^{\prime}}\right)$ and $\psi_{A} \rightarrow\left(\Psi_{a=1,2}, \Psi_{a^{\prime}=1^{\prime}, 2^{\prime}}\right)$, we obtain

$$
\begin{aligned}
S=\int \mathrm{d} t \mathrm{~d} x^{2}( & \kappa A_{0} F_{12}+\frac{\kappa}{2} \epsilon^{i j} \partial_{t} A_{i} A_{j}-\kappa \hat{A}_{0} \hat{F}_{12}-\frac{\kappa}{2} \epsilon^{i j} \partial_{t} \hat{A}_{i} \hat{A}_{j}+ \\
& +i \Phi_{A}^{*} D_{0} \Phi_{A}-\frac{1}{2} D_{i} \Phi_{A}^{*} D_{i} \Phi_{A}+ \\
& +i \Psi_{A}^{*} D_{0} \Psi_{A}-\frac{1}{2} D_{i} \Psi_{A}^{*} D_{i} \Psi_{A}- \\
& \left.-\frac{1}{2}\left(F_{12}-\hat{F}_{12}\right)\left(\Psi_{a}^{*} \Psi_{a}-\Psi_{a^{\prime}}^{*} \Psi_{a^{\prime}}\right)\right)
\end{aligned}
$$

We see that the action is equivalent to the non-relativistic ABJM model proposed in [15] in the Abelian case.

This superfield formulation reveals a hidden $S U(4)$ symmetry of the non-relativistic ABJM theory. The relativistic ABJM theory has an $S U(4)$ symmetry, while the mass deformation breaks it down to $S U(2) \times S U(2)$. As a consequence, only the $S U(2) \times$ $S U$ (2) symmetry has been manifest in the original construction of the non-relativistic ABJM theory. However, since we treat all $\Phi_{A}$ and $\Psi_{A}$ on the same footing, it is clear that the non-relativistic Abelian ABJM theory actually has an $S U(4)$ symmetry.

\section{Non-Abelian Gauge Theory}

It is straightforward to generalize the Abelian superfield formulation to the nonAbelian gauge theory. Again, the gauge multiplet consists of a vector superfield $\mathcal{V}$ 
and a chiral superfield $\mathcal{A}$ (and its conjugate $\mathcal{A}^{\dagger}$ ). They transform as adjoint representation of the gauge group $G$.

The gauge transformations are

$$
\begin{aligned}
\mathrm{e}^{\mathcal{V}} & \rightarrow \mathrm{e}^{\Lambda^{\dagger}} \mathrm{e}^{\mathcal{V}} \mathrm{e}^{\Lambda} \\
\mathrm{e}^{-\mathcal{V}} & \rightarrow \mathrm{e}^{-\Lambda^{-}} \mathrm{e}^{-\mathcal{V}} \mathrm{e}^{-\Lambda^{\dagger}} \\
\mathcal{A} & \rightarrow \mathrm{e}^{-\Lambda}\left(\mathcal{A}-2 i \partial_{+} \Lambda\right) \mathrm{e}^{\Lambda} \\
\mathcal{A}^{\dagger} & \rightarrow \mathrm{e}^{\Lambda^{\dagger}}\left(\mathcal{A}^{\dagger}+2 i \partial_{-} \Lambda^{\dagger}\right) \mathrm{e}^{-\Lambda^{\dagger}},
\end{aligned}
$$

where $\Lambda$ is an adjoint-valued chiral superfield. In the following, we work in the WZ-gauge where $\mathcal{V}=\theta \bar{\theta} 2 A_{0}$. The remaining transformation is the usual gauge transformation for the component fields.

The supersymmetric generalization of the non-Abelian Chern-Simons action is given by

$$
S_{\mathrm{CS}}=-\frac{\kappa}{16} \int \mathrm{d} t \mathrm{~d} x^{2} d \theta^{2} \operatorname{Tr}\left(\left(\mathcal{A}+2 i \partial_{+} \mathcal{V}\right) \mathrm{e}^{-\mathcal{V}}\left(\mathcal{A}^{\dagger}-2 i \partial_{-} \mathcal{V}\right) \mathrm{e}^{\mathcal{V}}-2\left(\partial_{+} \mathrm{e}^{\mathcal{V}}\right)\left(\partial_{-} \mathrm{e}^{-\mathcal{V}}\right)\right)
$$

The Lagrangian density is supersymmetric gauge invariant up to total derivative terms. In the component form, the action is given by

$$
S_{\mathrm{CS}}=\frac{\kappa}{2} \int \mathrm{d} t \mathrm{~d} x^{2}\left(\epsilon^{\mu \nu \rho} \operatorname{Tr}\left(A_{\mu} \partial_{\nu} A_{\rho}\right)+\frac{2 i}{3} \epsilon^{\mu \nu \rho} \operatorname{Tr}\left(A_{\mu} A_{\nu} A_{\rho}\right)-\frac{1}{8} \operatorname{Tr} \lambda \lambda^{\dagger}\right) .
$$

The matter multiplet (say, the fundamental representation) can be introduced by a chiral superfield $\Phi$ transforming as $\Phi \rightarrow \mathrm{e}^{-\Lambda} \Phi$ and associated fermionic chiral superfield $\Psi$ transforming as $\Psi \rightarrow \Psi \mathrm{e}^{\Lambda}$. We further impose the gauge covariant constraint

$$
D\left(\mathrm{e}^{\mathcal{V}} \Phi\right)=\partial_{-} \Psi^{\dagger}+\frac{i}{2} \mathcal{A}^{\dagger} \Psi^{\dagger}
$$

The simple matter action

$$
S_{\text {matter }}=-\frac{1}{2} \int \mathrm{d} t d^{2} x d^{2} \theta\left(\Phi^{\dagger} \mathrm{e}^{\mathcal{V}} \Phi-\Psi \mathrm{e}^{-\mathcal{V}} \Psi^{\dagger}\right)
$$

with the constraint (4.4) gives a non-Abelian generalization of the model discussed in Section 2.

\subsection{NON-ABELIAN NON-RELATIVISTIC ABJM}

As a final application, we would like to derive the non-Abelian non-relativistic ABJM theory in the superfield formulation so far developed in this paper. It has a manifest dynamical supersymmetry and we confirm the non-relativistic limit taken in [15] in a manifestly supersymmetric way. 
We first introduce $U(N) \times U(N)$ gauge connection with the gauge transformation

$$
\begin{aligned}
& \mathrm{e}^{\mathcal{V}} \rightarrow \mathrm{e}^{\Lambda^{\dagger}} \mathrm{e}^{\mathcal{V}} \mathrm{e}^{\Lambda} \\
& \mathrm{e}^{\hat{\mathcal{V}}} \rightarrow \mathrm{e}^{\hat{\Lambda}^{\dagger}} \mathrm{e}^{\hat{\mathcal{V}}} \mathrm{e}^{\hat{\Lambda}} \\
& \mathcal{A} \rightarrow \mathrm{e}^{-\Lambda}\left(\mathcal{A}-2 i \partial_{+} \Lambda\right) \mathrm{e}^{\Lambda} \\
& \hat{\mathcal{A}} \rightarrow \mathrm{e}^{-\hat{\Lambda}}\left(\hat{\mathcal{A}}-2 i \partial_{+} \hat{\Lambda}\right) \mathrm{e}^{\hat{\Lambda}} .
\end{aligned}
$$

The matter chiral multiplets $\left(\Phi_{a}, \Psi_{a}\right)$ and $\left(\Phi_{a^{\prime}}, \Psi_{a^{\prime}}\right)$ transform as

$$
\begin{aligned}
\Phi_{a} & \rightarrow \mathrm{e}^{-\hat{\Lambda}} \Phi_{a} \mathrm{e}^{\Lambda} \\
\Psi_{a} & \rightarrow \mathrm{e}^{-\Lambda} \Psi_{a} \mathrm{e}^{\hat{\Lambda}} \\
\Phi_{a^{\prime}} & \rightarrow \mathrm{e}^{-\Lambda} \Phi_{a^{\prime}} \mathrm{e}^{\hat{\Lambda}} \\
\Psi_{a^{\prime}} & \rightarrow \mathrm{e}^{-\hat{\Lambda}} \Psi_{a^{\prime}} \mathrm{e}^{\Lambda},
\end{aligned}
$$

where we have treated two indices $(a=1,2)$ and $\left(a^{\prime}=1^{\prime}, 2^{\prime}\right)$ differently, so there remains only $S U(2) \times S U(2)$ symmetry unlike the Abelian case in Section 3 .

The Chern-Simons part of the action is given by

$$
\begin{aligned}
S_{\mathrm{CS}}= & \int \mathrm{d} t \mathrm{~d} x^{2} \mathrm{~d} \theta^{2}-\frac{\kappa}{16} \operatorname{Tr}\left(\left(\mathcal{A}+2 i \partial_{+} \mathcal{V}\right) \mathrm{e}^{-\mathcal{V}}\left(\mathcal{A}^{\dagger}-2 i \partial_{-} \mathcal{V}\right) \mathrm{e}^{\mathcal{V}}-2\left(\partial_{+} \mathrm{e}^{\mathcal{V}}\right)\left(\partial_{-} \mathrm{e}^{-\mathcal{V}}\right)\right)+ \\
& +\frac{\kappa}{16} \operatorname{Tr}\left(\left(\hat{\mathcal{A}}+2 i \partial_{+} \hat{\mathcal{V}}\right) \mathrm{e}^{-\hat{\mathcal{V}}}\left(\hat{\mathcal{A}}^{\dagger}-2 i \partial_{-} \hat{\mathcal{V}}\right) \mathrm{e}^{\hat{\mathcal{V}}}-2\left(\partial_{+} \mathrm{e}^{\hat{\mathcal{V}}}\right)\left(\partial_{-} \mathrm{e}^{-\hat{\mathcal{V}}}\right)\right)- \\
= & \int \mathrm{d} t \mathrm{~d} x^{2} \frac{\kappa}{2}\left(\epsilon^{\mu \nu \rho} \operatorname{Tr}\left(A_{\mu} \partial_{\nu} A_{\rho}\right)+\frac{2 i}{3} \epsilon^{\mu \nu \rho} \operatorname{Tr}\left(A_{\mu} A_{\nu} A_{\rho}\right)-\frac{1}{8} \operatorname{Tr} \lambda \lambda^{\dagger}\right)- \\
& -\frac{\kappa}{2}\left(\epsilon^{\mu \nu \rho} \operatorname{Tr}\left(\hat{A}_{\mu} \partial_{\nu} \hat{A}_{\rho}\right)+\frac{2 i}{3} \epsilon^{\mu \nu \rho} \operatorname{Tr}\left(\hat{A}_{\mu} \hat{A}_{\nu} \hat{A}_{\rho}\right)-\frac{1}{8} \operatorname{Tr} \hat{\lambda} \hat{\lambda}^{\dagger}\right) .
\end{aligned}
$$

The matter action is given by the simple form

$$
\begin{aligned}
S_{\text {matter }}= & -\frac{1}{2} \int \mathrm{d} t \mathrm{~d} x^{2} \mathrm{~d} \theta^{2} \operatorname{Tr}\left(\Phi_{a}^{\dagger} \mathrm{e}^{\hat{\mathcal{V}}} \Phi_{a} \mathrm{e}^{-\mathcal{V}}+\Psi_{a}^{\dagger} \mathrm{e}^{\mathcal{V}} \Psi_{a} \mathrm{e}^{-\hat{\mathcal{V}}}+\right. \\
& \left.+\Phi_{a^{\prime}}^{\dagger} \mathrm{e}^{\mathcal{V}} \Phi_{a^{\prime}} \mathrm{e}^{-\hat{\mathcal{V}}}-\Psi_{a^{\prime}}^{\dagger} \mathrm{e}^{\hat{\mathcal{V}}} \Psi_{a^{\prime}} \mathrm{e}^{-\mathcal{V}}\right)
\end{aligned}
$$

with the following constraints:

$$
\begin{gathered}
D\left(\mathrm{e}^{\hat{\mathcal{V}}} \Phi_{a} \mathrm{e}^{-\mathcal{V}}\right)=\partial_{-} \Psi_{a}^{\dagger}+\frac{i}{2} \hat{\mathcal{A}}^{\dagger} \Psi_{a}^{\dagger}-\frac{i}{2} \Psi_{a}^{\dagger} \mathcal{A}^{\dagger} \\
D\left(\mathrm{e}^{\hat{\mathcal{V}}} \Psi_{a^{\prime}} \mathrm{e}^{-\mathcal{V}}\right)=\partial_{-} \Phi_{a^{\prime}}^{\dagger}+\frac{i}{2} \hat{\mathcal{A}}^{\dagger} \Phi_{a^{\prime}}^{\dagger}-\frac{i}{2} \Phi_{a^{\prime}}^{\dagger} \mathcal{A}^{\dagger}
\end{gathered}
$$


In components, we have

$$
\begin{aligned}
\chi_{a} & =\partial_{-} \psi_{a}^{\dagger}+i \hat{A}^{\dagger} \psi_{a}^{\dagger}-i \psi_{a}^{\dagger} A^{\dagger} \\
-2 i \partial_{t} \phi_{a}+2 \hat{A}_{0} \phi_{a}-2 \phi_{a} A_{0} & =\partial_{-} \rho_{a}^{\dagger}+i \hat{A}^{\dagger} \rho_{a}^{\dagger}-i \rho_{a}^{\dagger} A^{\dagger}-\frac{i}{2} \hat{\lambda}^{\dagger} \psi_{a}^{\dagger}+\frac{i}{2} \psi_{a}^{\dagger} \lambda^{\dagger} \\
\rho_{a^{\prime}} & =\partial_{-} \phi_{a^{\prime}}^{\dagger}+i \hat{A}^{\dagger} \phi_{a^{\prime}}^{\dagger}-i \phi_{a^{\prime}}^{\dagger} A^{\dagger} \\
-2 i \partial_{t} \psi_{a^{\prime}}+2 \hat{A}_{0} \psi_{a^{\prime}}-2 \psi_{a^{\prime}} A_{0} & =-\partial_{-} \chi_{a^{\prime}}^{\dagger}-i \hat{A}^{\dagger} \chi_{a^{\prime}}^{\dagger}+i \chi_{a^{\prime}}^{\dagger} A^{\dagger}-\frac{i}{2} \hat{\lambda}^{\dagger} \phi_{a^{\prime}}^{\dagger}+\frac{i}{2} \phi_{a^{\prime}}^{\dagger} \lambda^{\dagger}
\end{aligned}
$$

The matter Lagrangian with the constraints imposed by the Lagrange multipliers is written as

$$
\begin{aligned}
& \operatorname{Tr}\left(i \phi_{a}^{\dagger} D_{t} \phi_{a}-\frac{1}{2} D_{-} \psi_{a}^{\dagger} D_{+} \psi_{a}+i \psi_{a}^{\dagger} D_{t} \psi_{a}+\frac{1}{2} \rho_{a}^{\dagger} \rho_{a}+\right. \\
& \quad+c_{a}\left(i D_{t} \phi_{a}+\frac{1}{2} D_{-} \rho_{a}^{\dagger}-\frac{i}{4} \hat{\lambda}^{\dagger} \psi_{a}^{\dagger}+\frac{i}{4} \psi_{a}^{\dagger} \lambda^{\dagger}\right)+\left(-i D_{t} \phi_{a}^{\dagger}+\frac{1}{2} D_{+} \rho_{a}+\right. \\
& \left.\quad+\frac{i}{4} \psi_{a} \hat{\lambda}-\frac{i}{4} \lambda \psi_{a}\right) c_{a}^{\dagger}+i \psi_{a^{\prime}}^{\dagger} D_{t} \psi_{a^{\prime}}+\frac{1}{2} \chi_{a^{\prime}}^{\dagger} \chi_{a^{\prime}}-i \psi_{a^{\prime}}^{\dagger} D_{t} \psi_{a^{\prime}}-\frac{1}{2} D_{-} \phi_{a^{\prime}}^{\dagger} D_{+} \phi_{a^{\prime}}+ \\
& \quad+\xi_{a^{\prime}}\left(i D_{t} \psi_{a^{\prime}}-\frac{1}{2} D_{-} \chi_{a^{\prime}}^{\dagger}-\frac{i}{4} \hat{\lambda}^{\dagger} \phi_{a^{\prime}}^{\dagger}+\frac{i}{4} \phi_{a^{\prime}}^{\dagger} \lambda^{\dagger}\right) \\
& \left.\quad+\left(-i D_{t} \psi_{a^{\prime}}^{\dagger}-\frac{1}{2} D_{+} \chi_{a^{\prime}}+\frac{i}{4} \phi_{a^{\prime}} \hat{\lambda}-\frac{i}{4} \lambda \phi_{a^{\prime}}\right) \xi_{a^{\prime}}^{\dagger}\right)
\end{aligned}
$$

where $c_{a}$ are bosonic Lagrange multipliers and $\xi_{a^{\prime}}$ are fermionic ones. We can eliminate the auxiliary fields $\lambda, \hat{\lambda}, \rho_{a}, \chi_{a^{\prime}}$ as well as $\phi_{a}, \psi_{a^{\prime}}$ as

$$
\begin{aligned}
\frac{\kappa}{16} \hat{\lambda}+\frac{i}{4} \psi_{a}^{\dagger} c_{a}-\frac{i}{4} \phi_{a^{\prime}}^{\dagger} \xi_{a^{\prime}} & =0 \\
-\frac{\kappa}{16} \lambda+\frac{i}{4} c_{a} \psi_{a}^{\dagger}+\frac{i}{4} \xi_{a^{\prime}} \phi_{a^{\prime}}^{\dagger} & =0 \\
\rho_{a}-D_{-} c_{a} & =0 \\
\chi_{a^{\prime}}-D_{-} \xi_{a^{\prime}} & =0 \\
i D_{t} \phi_{a}+i D_{t} c_{a}^{\dagger} & =0 \\
-i D_{t} \psi_{a^{\prime}}+i D_{t} \xi_{a^{\prime}}^{\dagger} & =0
\end{aligned}
$$

Substituting them back into the matter Lagrangian, we obtain the matter Lagrangian:

$$
\begin{aligned}
& \operatorname{Tr}\left(i c_{a}^{\dagger} D_{t} c_{a}-\frac{1}{2} D_{+} c_{a}^{\dagger} D_{-} c_{a}+i \psi_{a}^{\dagger} D_{t} \psi_{a}-\frac{1}{2} D_{-} \psi_{a}^{\dagger} D_{+} \psi_{a}+\right. \\
& \quad+i \phi_{a^{\prime}}^{\dagger} D_{t} \phi_{a^{\prime}}-\frac{1}{2} D_{-} \phi_{a^{\prime}}^{\dagger} D_{+} \phi_{a^{\prime}}+i \xi_{a^{\prime}}^{\dagger} D_{t} \xi_{a^{\prime}}-\frac{1}{2} D_{+} \xi_{a^{\prime}}^{\dagger} D_{-} \xi_{a^{\prime}}- \\
& \left.\quad-\frac{1}{\kappa}\left(\psi_{a}^{\dagger} c_{a}-\phi_{a^{\prime}}^{\dagger} \xi_{a^{\prime}}\right)\left(c_{b}^{\dagger} \psi_{b}-\xi_{b^{\prime}}^{\dagger} \phi_{b^{\prime}}\right)+\frac{1}{\kappa}\left(c_{a} \psi_{a}^{\dagger}-\xi_{a^{\prime}} \phi_{a^{\prime}}^{\dagger}\right)\left(\psi_{a} c_{a}^{\dagger}-\phi_{a^{\prime}} \xi_{a^{\prime}}^{\dagger}\right)\right)
\end{aligned}
$$


In order to compare it with the one presented in [15], we use the Gauss-law constraint

$$
\begin{aligned}
& F_{12}=\frac{1}{\kappa}\left(c_{a} c_{a}^{\dagger}-\psi_{a} \psi_{a}^{\dagger}+\phi_{a^{\prime}} \phi_{a^{\prime}}^{\dagger}-\xi_{a^{\prime}} \xi_{a^{\prime}}^{\dagger}\right) \\
& \hat{F}_{12}=\frac{1}{\kappa}\left(c_{a}^{\dagger} c_{a}+\psi_{a}^{\dagger} \psi_{a}+\phi_{a^{\prime}}^{\dagger} \phi_{a^{\prime}}+\xi_{a^{\prime}}^{\dagger} \xi_{a^{\prime}}\right)
\end{aligned}
$$

to rewrite the kinetic term for scalars as

$$
\begin{aligned}
-\frac{1}{2} \operatorname{Tr}\left(D_{+} c_{a}^{\dagger} D_{-} c_{a}\right)= & -\frac{1}{2} \operatorname{Tr}\left(D_{i} c_{a}^{\dagger} D_{i} c_{a}\right)-\frac{1}{2} \operatorname{Tr}\left(c_{a}^{\dagger} c_{a} \hat{F}_{12}\right)+\frac{1}{2} \operatorname{Tr}\left(c_{a}^{\dagger} F_{12} c_{a}\right)= \\
= & -\frac{1}{2} \operatorname{Tr}\left(D_{i} c_{a}^{\dagger} D_{i} c_{a}\right)-\frac{1}{2 \kappa} \operatorname{Tr}\left(c_{a}^{\dagger} c_{a}\left(c_{b}^{\dagger} c_{b}+\psi_{b}^{\dagger} \psi_{b}+\phi_{b^{\prime}}^{\dagger} \phi_{b^{\prime}}+\xi_{b^{\prime}}^{\dagger} \xi_{b^{\prime}}\right)\right)+ \\
& +\frac{1}{2 \kappa} \operatorname{Tr}\left(c_{a}^{\dagger}\left(c_{b} c_{b}^{\dagger}-\psi_{b} \psi_{b}^{\dagger}+\phi_{b^{\prime}} \phi_{b^{\prime}}^{\dagger}-\xi_{b^{\prime}} \xi_{b^{\prime}}^{\dagger}\right) c_{a}\right) \\
-\frac{1}{2} \operatorname{Tr}\left(D_{-} \phi_{a^{\prime}}^{\dagger} D_{+} \phi_{a^{\prime}}\right)= & -\frac{1}{2} \operatorname{Tr}\left(D_{i} \phi_{a^{\prime}}^{\dagger} D_{i} \phi_{a^{\prime}}\right)+\frac{1}{2} \operatorname{Tr}\left(\phi_{a^{\prime}}^{\dagger} \phi_{a^{\prime}} \hat{F}_{12}\right)-\frac{1}{2} \operatorname{Tr}\left(\phi_{a^{\prime}}^{\dagger} F_{12} \phi_{a^{\prime}}\right)= \\
= & -\frac{1}{2} D_{i} \phi_{a^{\prime}}^{\dagger} D_{i} \phi_{a^{\prime}}^{\dagger}+\frac{1}{2 \kappa} \operatorname{Tr}\left(\phi_{a^{\prime}}^{\dagger} \phi_{a^{\prime}}\left(c_{b}^{\dagger} c_{b}+\psi_{b}^{\dagger} \psi_{b}+\phi_{b^{\prime}}^{\dagger} \phi_{b^{\prime}}+\xi_{b^{\prime}}^{\dagger} \xi_{b^{\prime}}\right)\right)- \\
& -\frac{1}{2 \kappa} \operatorname{Tr}\left(\phi_{a^{\prime}}^{\dagger}\left(c_{b} c_{b}^{\dagger}-\psi_{b} \psi_{b}^{\dagger}+\phi_{b^{\prime}} \phi_{b^{\prime}}^{\dagger}-\xi_{b^{\prime}} \xi_{b^{\prime}}^{\dagger}\right) \phi_{a^{\prime}}\right)
\end{aligned}
$$

Note that the scalar potential solely comes from this rewriting and it is summarized as

$$
V_{\mathrm{bos}}=-\frac{1}{2 \kappa} \operatorname{Tr}\left(\phi_{a} \phi_{[a}^{\dagger} \phi_{b} \phi_{b]}^{\dagger}-\phi_{a^{\prime}} \phi_{\left[a^{\prime}\right.}^{\dagger} \phi_{b^{\prime}} \phi_{\left.b^{\prime}\right]}^{\dagger}\right),
$$

where we have renamed $c_{a} \rightarrow i \phi_{a}$ in order to adjust to the convention used in [15].

Finally, we rename the fermionic fields: $\psi_{a} \rightarrow i \epsilon_{a b} \psi_{b}, \xi_{a^{\prime}} \rightarrow i \epsilon_{a^{\prime} b^{\prime}} \psi_{b^{\prime}}$. With this renaming, the action completely agrees with that in [15] (with the replacement $k=2 \pi \kappa$ and $m=1)$ :

$$
S_{\mathrm{ABJM}}=\int \mathrm{d} t d^{2} x\left(L_{\mathrm{CS}}+L_{\mathrm{kin}}-V_{\mathrm{bos}}-V_{\mathrm{fer}}\right),
$$

where

$$
\begin{aligned}
L_{\mathrm{CS}}= & \frac{\kappa}{2}\left(\epsilon^{\mu \nu \rho} \operatorname{Tr}\left(A_{\mu} \partial_{v} A_{\rho}\right)+\frac{2 i}{3} \epsilon^{\mu \nu \rho} \operatorname{Tr}\left(A_{\mu} A_{v} A_{\rho}\right)-\epsilon^{\mu \nu \rho} \operatorname{Tr}\left(\hat{A}_{\mu} \partial_{v} \hat{A}_{\rho}\right)-\right. \\
& \left.\quad-\frac{2 i}{3} \epsilon^{\mu \nu \rho} \operatorname{Tr}\left(\hat{A}_{\mu} \hat{A}_{v} \hat{A}_{\rho}\right)\right) \\
L_{\mathrm{kin}}= & \operatorname{Tr}\left(i \phi_{a}^{\dagger} D_{t} \phi_{a}-\frac{1}{2} D_{i} \phi_{a}^{\dagger} D_{i} \phi_{a}+i \psi_{a}^{\dagger} D_{t} \psi_{a}-\frac{1}{2} D_{-} \psi_{a}^{\dagger} D_{+} \psi_{a}+\right. \\
& \left.+i \phi_{a^{\prime}}^{\dagger} D_{t} \phi_{a^{\prime}}-\frac{1}{2} D_{i} \phi_{a^{\prime}}^{\dagger} D_{i} \phi_{a^{\prime}}+i \psi_{a^{\prime}}^{\dagger} D_{t} \psi_{a^{\prime}}-\frac{1}{2} D_{+} \psi_{a^{\prime}}^{\dagger} D_{-} \psi_{a^{\prime}}\right) \\
V_{\mathrm{bos}}= & -\frac{1}{2 \kappa} \operatorname{Tr}\left(\phi_{a} \phi_{[a}^{\dagger} \phi_{b} \phi_{b]}^{\dagger}-\phi_{a^{\prime}} \phi_{\left[a^{\prime}\right.}^{\dagger} \phi_{b^{\prime}} \phi_{\left.b^{\prime}\right]}^{\dagger}\right)
\end{aligned}
$$




$$
\begin{aligned}
V_{\mathrm{fer}}= & -\frac{1}{2 \kappa} \operatorname{Tr}\left[\left(\phi_{a}^{\dagger} \phi_{a}+\phi_{a^{\prime}}^{\dagger} \phi_{a^{\prime}}\right)\left(\psi_{b}^{\dagger} \psi_{b}-\psi_{b^{\prime}}^{\dagger} \psi_{b^{\prime}}\right)+\left(\phi_{a} \phi_{a}^{\dagger}+\phi_{a^{\prime}} \phi_{a^{\prime}}^{\dagger}\right)\left(\psi_{b} \psi_{b}^{\dagger}-\psi_{b^{\prime}} \psi_{b^{\prime}}^{\dagger}\right)-\right. \\
& -2 \phi_{a} \phi_{b}^{\dagger} \psi_{a} \psi_{b}^{\dagger}+2 \phi_{a^{\prime}} \phi_{b^{\prime}}^{\dagger} \psi_{a^{\prime}} \psi_{b^{\prime}}^{\dagger}-2 \phi_{a}^{\dagger} \phi_{b} \psi_{a}^{\dagger} \psi_{b}+2 \phi_{a^{\prime}}^{\dagger} \phi_{b^{\prime}} \psi_{a^{\prime}}^{\dagger} \psi_{b^{\prime}}- \\
& -i \epsilon^{a b} \epsilon^{c^{\prime} d^{\prime}} \phi_{a}^{\dagger} \psi_{b} \phi_{c^{\prime}}^{\dagger} \psi_{d^{\prime}}-i \epsilon^{b c} \epsilon^{a^{\prime} d^{\prime}} \phi_{a^{\prime}}^{\dagger} \psi_{b} \phi_{c}^{\dagger} \psi_{d^{\prime}}+i \epsilon^{a^{\prime} b^{\prime}} \epsilon^{c d} \phi_{a^{\prime}}^{\dagger} \psi_{b^{\prime}} \phi_{c}^{\dagger} \psi_{d}- \\
& +i \epsilon^{b^{\prime} c^{\prime}} \epsilon^{a d} \phi_{a}^{\dagger} \psi_{b^{\prime}} \phi_{c^{\prime}}^{\dagger} \psi_{d}+i \epsilon^{a b} \epsilon^{c^{\prime} d^{\prime}} \phi_{a} \psi_{b}^{\dagger} \phi_{c^{\prime}} \psi_{d^{\prime}}^{\dagger}+i \epsilon^{b c} \epsilon^{a^{\prime} d^{\prime}} \phi_{a^{\prime}} \psi_{b}^{\dagger} \phi_{c} \psi_{d^{\prime}}^{\dagger}- \\
& \left.-i \epsilon^{a^{\prime} b^{\prime}} \epsilon^{c d} \phi_{a^{\prime}} \psi_{b^{\prime}}^{\dagger} \phi_{c} \psi_{d}^{\dagger}-i \epsilon^{b^{\prime} c^{\prime}} \epsilon^{a d} \phi_{a} \psi_{b^{\prime}}^{\dagger} \phi_{c^{\prime}} \psi_{d}^{\dagger}\right]
\end{aligned}
$$

\section{Discussions}

In this paper, we have developed a superfield formulation for non-relativistic Chern-Simons-matter theories in $(1+2)$ dimension. We have successfully reproduced the non-relativistic Chern-Simons-matter theories proposed in the literatures while manifestly preserving the dynamical supersymmetry.

Our formulation is minimal in the sense that it only possesses manifest dynamical supersymmetry, which has both advantage and disadvantage. The disadvantage is that we fail in manifesting some additional symmetries such as Poincare invariance or Galilean invariance. On the other hand, this minimal structure allows us to study the theory with no such additional structures. For instance, we can easily introduce the supersymmetric extensions of the Maxwell term by adding $S_{E}+S_{M}$ with

$$
\begin{aligned}
S_{\mathrm{E}} & =\frac{1}{16 g_{E}^{2}} \int \mathrm{d} t d^{2} x d^{2} \theta\left(D\left(\mathcal{A}+2 i \partial_{+} \mathcal{V}\right) \bar{D}\left(\mathcal{A}^{*}-2 i \partial_{-} \mathcal{V}\right)\right) \\
S_{\mathrm{M}} & =-\frac{1}{2 g_{M}^{2}} \int \mathrm{d} t d^{2} x d^{2} \theta \Gamma^{*} \Gamma
\end{aligned}
$$

where we impose the constraint for a fermionic chiral multiplet $\Gamma$ as

$$
D \Gamma+\bar{D} \Gamma^{*}=-i\left(\partial_{-} \mathcal{A}-\partial_{+} \mathcal{A}^{*}+2 i \partial_{-} \partial_{+} \mathcal{V}\right) .
$$

Similarly, we could study the supersymmetric extension of the Hor̆ava-Lifshitz term, where we need two additional fermionic chiral multiplets $\Gamma_{i}$ with the constraint $^{6}$

$$
D \Gamma_{i}+\bar{D} \Gamma_{i}^{*}=-i \partial_{i}\left(\partial_{-} \mathcal{A}-\partial_{+} \mathcal{A}^{*}+2 i \partial_{-} \partial_{+} \mathcal{V}\right) .
$$

The corresponding action is

$$
S_{\mathrm{HL}}=-\alpha \int \mathrm{d} t d^{2} x d^{2} \theta \Gamma_{i}^{*} \Gamma_{i} .
$$

In this paper, we have shown the manifestly supersymmetric form of the nonrelativistic ABJM theory with 14 supercharges. We can study different (less supersymmetric) non-relativistic limits of the ABJM model presented in [15]. In order to

\footnotetext{
${ }^{6}$ As a consequence, the "gaugino" $\zeta_{i}=\left.\partial_{\theta} \Gamma_{i}\right|_{\theta=0}$ has a vector indices $i=1,2$. This has been independently observed by C. M. Thompson.
} 
realize different limits in our superfield approach, we simply change the constraint for $\left(a^{\prime}\right)$ multiplets: $D\left(\mathrm{e}^{\mathcal{V}} \Phi_{a^{\prime}} \mathrm{e}^{-\hat{\mathcal{V}}}\right)=\partial_{-} \Psi_{a}^{\dagger}+\frac{i}{2} \mathcal{A}^{\dagger} \Psi_{a^{\prime}}^{\dagger}-\frac{i}{2} \Psi_{a^{\prime}}^{\dagger} \hat{\mathcal{A}}^{\dagger}$. The matter content is exactly the same as that studied in section 4 of [15]:

$$
\begin{aligned}
L_{\mathrm{kin}}= & \operatorname{Tr}\left(i \phi_{a}^{\dagger} D_{t} \phi_{a}-\frac{1}{2} D_{+} \phi_{a}^{\dagger} D_{-} \phi_{a}+i \psi_{a}^{\dagger} D_{t} \psi_{a}-\frac{1}{2} D_{-} \psi_{a}^{\dagger} D_{+} \psi_{a}+\right. \\
& \left.+i \hat{\phi}_{a^{\prime}}^{\dagger} D_{t} \hat{\phi}_{a^{\prime}}-\frac{1}{2} D_{+} \hat{\phi}_{a^{\prime}}^{\dagger} D_{-} \hat{\phi}_{a^{\prime}}+i \hat{\psi}_{a^{\prime}}^{\dagger} D_{t} \hat{\psi}_{a^{\prime}}-\frac{1}{2} D_{-} \hat{\psi}_{a^{\prime}}^{\dagger} D_{+} \hat{\psi}_{a^{\prime}}\right)
\end{aligned}
$$

However, we note that the potential is different: ${ }^{7}$

$$
V=\frac{1}{\kappa}\left(\psi_{a}^{\dagger} \phi_{a}+\hat{\phi}_{a^{\prime}} \hat{\psi}_{a^{\prime}}^{\dagger}\right)\left(\phi_{a}^{\dagger} \psi_{a}+\hat{\psi}_{a^{\prime}} \hat{\phi}_{a^{\prime}}^{\dagger}\right)-\frac{1}{\kappa}\left(\phi_{a} \psi_{a}^{\dagger}+\hat{\psi}_{a^{\prime}}^{\dagger} \hat{\phi}_{a^{\prime}}\right)\left(\psi_{a} \phi_{a}^{\dagger}+\hat{\phi}_{a^{\prime}}^{\dagger} \hat{\psi}_{a^{\prime}}\right)
$$

Actually, there is another possibility: we exchange the representation of $\Phi_{a^{\prime}}$ with that of $\Psi_{a^{\prime}}$ and introduce the constraint $D\left(\mathrm{e}^{\mathcal{V}} \Psi_{a^{\prime}} \mathrm{e}^{-\hat{\mathcal{V}}}\right)=\partial_{-} \Phi_{a^{\prime}}^{\dagger}+\frac{i}{2} \mathcal{A}^{\dagger} \Phi_{a^{\prime}}^{\dagger}-\frac{i}{2} \Phi_{a^{\prime}}^{\dagger} \hat{\mathcal{A}}^{\dagger}$. This gives a different result, but it again shows potential terms that do not arise in the non-relativistic limit of ABJM theory.

All these are consistent because it was shown that the non-relativistic limit taken in section 4 of [15] only preserves the kinematical supersymmetry. The superfield formulation here shows that there exists a deformation of the potential so that the dynamical supersymmetry is preserved. Incidentally, it is this deformed non-relativistic ABJM theory whose index was computed in [13]. It would be interesting to give clear physical understanding of this deformation from the viewpoint of the original ABJM theory.

\section{Acknowledgements}

The author would like to thank M. Sakaguchi, C. M. Thompson and K. Yoshida for fruitful discussions. The work was supported in part by the National Science Foundation under Grant No. PHY05-55662 and the UC Berkeley Center for Theoretical Physics.

Open Access This article is distributed under the terms of the Creative Commons Attribution Noncommercial License which permits any noncommercial use, distribution, and reproduction in any medium, provided the original author(s) and source are credited.

\section{References}

1. Aharony, O., Bergman, O., Jafferis, D.L., Maldacena, J.: $\mathrm{N}=6$ superconformal ChernSimons-matter theories, M2-branes and their gravity duals. JHEP 0810, 091 (2008) [arXiv:0806.1218 [hep-th]]

${ }^{7}$ For example, the terms like $\epsilon^{a b} \epsilon^{c^{\prime} d^{\prime}} \phi_{a}^{\dagger} \psi_{b} \hat{\phi}_{c^{\prime}}^{\dagger} \hat{\psi}_{d^{\prime}}$ are missing in the action presented in Section 4.2 of [15] even if we rewrite the potential by using the Gauss-law constraint. This was independently pointed out by M. Sakaguchi. 
2. Balasubramanian, K., McGreevy, J.: Gravity duals for non-relativistic CFTs. arXiv:0804.4053 [hep-th]

3. Barut, A.O., Xu, B.W.: Conformal covariance and the probability interpretation of wave equations. Phys. Lett. A 82, 218 (1981)

4. de Azcarraga, J.A., Ginestar, D.: Nonrelativistic limit of supersymmetric theories. J. Math. Phys. 32, 3500 (1991)

5. Gates, S.J., Grisaru, M.T., Rocek, M., Siegel, W.: Superspace, or one thousand and one lessons in supersymmetry. Front. Phys. 58, 1 (1983) [arXiv:hep-th/0108200]

6. Gomis, J., Rodriguez-Gomez, D., Van Raamsdonk, M., Verlinde, H.: A massive study of M2-brane proposals. JHEP 0809, 113 (2008) [arXiv:0807.1074 [hep-th]]

7. Hagen, C.R.: Scale and conformal transformations in galilean-covariant field theory. Phys. Rev. D 5, 377 (1972)

8. Horava, P.: Quantum criticality and Yang-Mills gauge theory. arXiv:0811.2217 [hep-th]

9. Hosomichi, K., Lee, K.M., Lee, S., Lee, S., Park, J.: $\mathrm{N}=5,6$ superconformal ChernSimons theories and M2-branes on orbifolds. JHEP 0809, 002 (2008) [arXiv:0806.4977 [hep-th]]

10. Jackiw, R., Pi, S.Y.: Classical and quantal nonrelativistic Chern-Simons theory. Phys. Rev. D 42, 3500 (1990) [Erratum-ibid. D 48, 3929 (1993)]

11. Jackiw, R., Pi, S.Y.: Finite and infinite symmetries in $(2+1)$-dimensional field theory. arXiv:hep-th/9206092

12. Leblanc, M., Lozano, G., Min, H.: Extended superconformal Galilean symmetry in Chern-Simons matter systems. Ann. Phys. 219, 328 (1992) [arXiv:hep-th/9206039]

13. Nakayama, Y.: Index for Non-relativistic Superconformal field theories. JHEP 0810, 083 (2008) [arXiv:0807.3344 [hep-th]].

14. Nakayama, Y., Sakaguchi, M., Yoshida, K.: Interacting SUSY-singlet matter in non-relativistic Chern-Simons theory. arXiv:0812.1564 [hep-th].

15. Nakayama, Y., Sakaguchi, M., Yoshida, K.: Non-Relativistic M2-brane gauge theory and new superconformal algebra. arXiv:0902.2204 [hep-th]

16. Nakayama, Y., Ryu, S., Sakaguchi, M., Yoshida, K.: A family of super Schrodinger invariant Chern-Simons matter systems. JHEP 0901, 006 (2009) [arXiv:0811.2461 [hep-th]]

17. Nicolai, H.: Supersymmetry and spin systems. J. Phys. A 9, 1497 (1976)

18. Niederer, U.: The maximal kinematical invariance group of the free Schrodinger equation. Helv. Phys. Acta 45, 802 (1972)

19. Perroud, M.: Projective representations of the Schrodinger Group. Helv. Phys. Acta 50, 233 (1977)

20. Salam, A., Strathdee, J.A.: Supergauge transformations. Nucl. Phys. B 76, 477 (1974)

21. Son, D.T.: Toward an AdS/cold atoms correspondence: a geometric realization of the Schroedinger symmetry. arXiv:0804.3972 [hep-th]

22. Wess, J., Bagger, J.: Supersymmetry and supergravity. Princeton University Press, Princeton, p. 259 (1992) 